\title{
Abexinostat Tosylate
}

\author{
National Cancer Institute
}

\section{Source}

National Cancer Institute. Abexinostat Tosylate. NCI Thesaurus. Code C160889.

The tosylate salt form of abexinostat, an orally bioavailable hydroxamate-based paninhibitor of histone deacetylase (HDAC), with potential antineoplastic and radiosensitizing activities. Upon administration, abexinostat inhibits HDAC, resulting in an accumulation of highly acetylated histones, followed by the induction of chromatin remodeling; the selective transcription of tumor suppressor genes; and the tumor suppressor proteinmediated inhibition of tumor cell division and induction of tumor cell apoptosis. In addition, abexinostat decreases the expression of the DNA-repair protein RAD51, thereby reducing the RAD51 protein, preventing repair of DNA double-strand breaks and increasing sensitivity of tumor cells to DNA damaging agents. HDAC, upregulated in many tumor types, is an enzyme that is responsible for the deacetylation of chromatin histone proteins. 